\title{
Performance Evaluation of Proposed Segmentation Framework with Existing Techniques for Noisy Iris Images
}

\author{
Rajeev Gupta \\ Department of Computer Applications \\ Maharishi Markandeshwar University, Mullana \\ (Ambala), Haryana (India)
}

\author{
Ashok Kumar \\ Department of Computer Engineering \\ Maharishi Markandeshwar University, Mullana \\ (Ambala), Haryana (India)
}

\begin{abstract}
While different iris segmentation techniques continue to appear, there has been a lack of recognition accuracy of existing methods with noisy iris dataset. To handle iris images (captured on less constrained conditions) with some types of noise (iris obstructions and specular reflection), the authors shows the results of performance evaluation of their proposed iris segmentation technique over existing techniques. The performance of a proposed iris segmentation technique is evaluated based on the accuracy and time. To evaluate the performance, the authors use the most important points to compare their proposed technique with others, which is Equal Error Rate (EER). The system is implemented and tested using MATLAB Version 7.5.0.342 (R2007b) software. The environment where the experiments are performed in is Compaq PC, Core 2 Due Intel Pentium Processor $(2.00 \mathrm{GHz})$, with $1 \mathrm{~GB}$ RAM and Windows 7 operating system, a dataset of UBIRIS v1, UBIRIS v2 and CASIA-IrisV4 databases samples of iris data with different contrast quality.
\end{abstract}

\section{General Terms}

Biometrics, Image Processing, Iris Segmentation

\section{Keywords}

Noisy Iris Dataset, Specular Reflection, Edge Detection, Iris Obstructions, Upper Eyelid

\section{INTRODUCTION}

Iris segmentation is a very crucial step for initial stage of iris recognition system. For iris segmentation, many researchers have contributed their efforts \& used different techniques to increase the performance of iris recognition system. Basically, iris segmentation techniques can be classified into two categories (see Figure 1). The iris segmentation stage is important because it is the base step of all further operations, such as Iris Normalization, Feature Extraction and Feature Comparison. There are already many iris segmentation techniques proposed by various researchers. But, these techniques fail when dealing with noisy iris images. The accuracy of existing iris segmentation techniques is significantly decreases when dealing with noisy iris images available in UBIRIS v1, UBIRIS v2 and CASIA-IrisV4 databases. The proposed segmentation technique[1][2] handles noisy iris images captured in less constrained conditions and consists the noise such as iris obstructions and specular reflection This paper summarizes the performance evaluation of proposed iris segmentation framework with existing techniques for noisy iris images for a secure iris recognition system. Part 2 focuses on the related work; Part 3 presents the common public and freely available iris image databases; Part 4 focuses on performance evaluation of proposed framework; and Part 5 presents the conclusion and future scope.

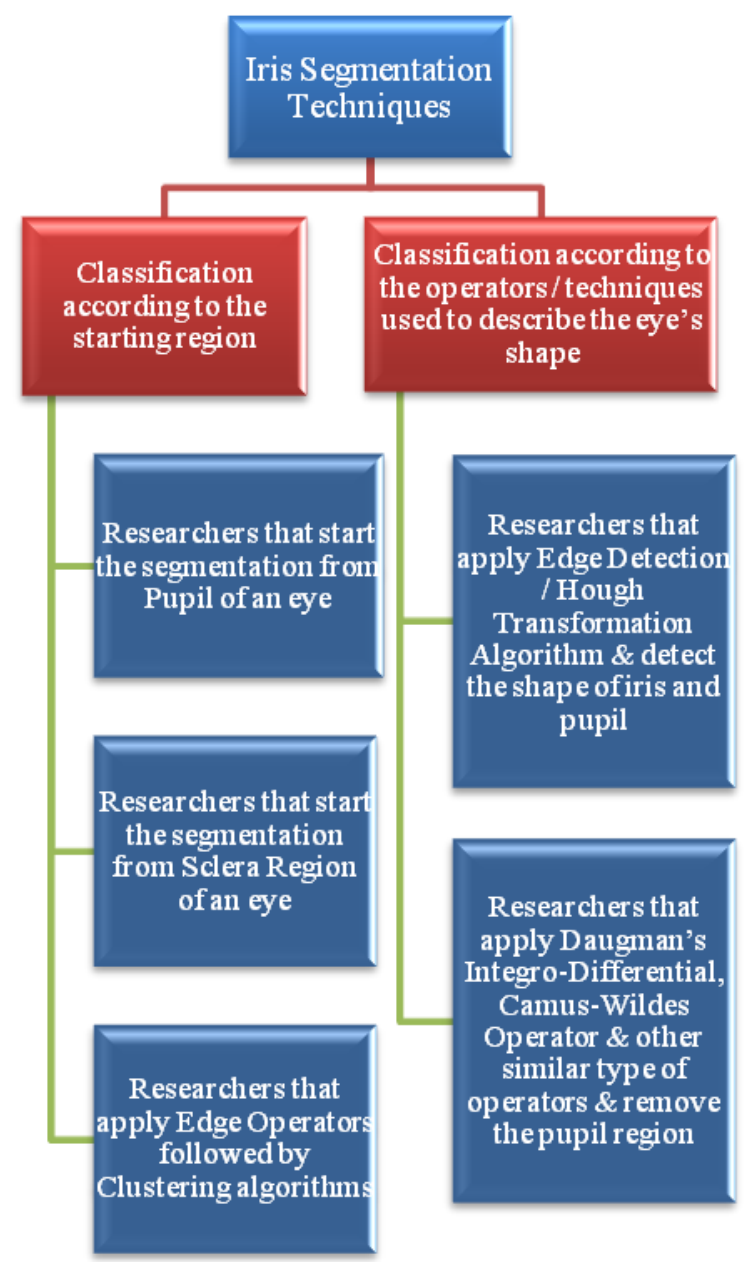

Figure 1 Iris Segmentation Techniques

\section{RELATED WORK}

The most famous and robust iris segmentation methods for noisy image dataset are as follows:

Daugman's technique proposed in 1993, is the most popular and cited iris segmentation technique and is licensed to Iridian Technologies on the basis of their $99.5 \%$ usage in the commercial iris recognition systems[3]. In this technique, Daugman assumes both pupil and iris with circular form and applies the following operator 


$$
\max _{r, x 0, y 0}\left|G_{\sigma}(r) * \frac{\delta}{\delta r} \oint_{r, x 0, y 0} \frac{I(x, y)}{2 \pi r} d s\right|
$$

Here, $I(x, y)$ is an image; $d s$ is circular arc of radius $\mathrm{r}$; $(x 0, y 0)$ are Center coordinates; Symbol * denotes convolution; and $G_{\sigma}(r)$ is a smoothing function. This process works very effective on images with clear intensity separately between iris, pupil and sclera regions. But the major disadvantage of this method is that it frequently fails when the images do not have sufficient intensity separately between iris and sclera regions and also fails where there is existence of some type of noise in the eye image, such as reflections. It works only on images picked at Near Infrared camera and in ideal imaging conditions.

Camus and Wildes proposed a technique for localizing the iris and pupil boundaries by using multi-resolution approach of an eye in a close-up image [4]. This technique used both the pupil and iris boundaries as a component goodness-of-fit metric for boundary parameters in a polar coordinate system. The component-goodness-of-fit is defined as

$$
C=\sum_{\theta=1}^{n}\left((n-1)\left\|g_{\theta, r}\right\|-\sum_{\phi=\theta+1}^{n}\left(\left\|g_{\theta, r}-g_{\phi, r}\right\|\right)-\frac{I_{\theta, r}}{n}\right)
$$

where $n$ is the total number of directions and $I_{\theta, r}$ and $g_{\theta, r}$ are respectively the image intensity and derivatives with respect to the radius in the polar coordinate system. But, the major disadvantage of this approach that it shows declined accuracy when dealing with noise effected data [5]

Proenca also proposed a technique to segment noisy images acquired in the visible wavelength [6] by using the flow of criteria of detecting the sclera, iris and noise-free iris regions. This method is very accurate with noisy images that taken in visible wavelength. But, major limitation of this method is to determine the region of iris when the sclera covered with dark color caused by bad image picked environments or eye diseases.

Martin-Roche methodology [7], works in a similar to Daugman's methodology. It receives a grey-scale image, applies the histogram stretch and tries to maximize the average intensity differences of the five consecutive circumferences, defined as

$$
D=\sum_{m}\left(\sum_{k=1}^{5}\left(I_{n, m}-I_{n-k, m}\right)\right)
$$

Where

$I_{i, j}=I\left(x_{0}+i \Delta_{r} \cos \left(j \Delta_{\Theta}\right), y_{0}+i \Delta_{r} \sin \left(j \Delta_{\Theta}\right)\right)$.

$\Delta_{r}$ and $\Delta_{\Theta}$ are the increments of radius and angle, respectively, and $\mathrm{I}(\mathrm{x}, \mathrm{y})$ is the image intensity.

\section{PUBLIC NOISY IRIS IMAGE DATABASES}

In this section, the authors describe the common public and freely available iris image databases, which will be used in the research. There are many iris image databases freely such as CASIA, UBIRIS, MMU (Multimedia University, 2004), ICE (National Institute of Standards and Technology, 2006) and UPOL [8]. Here, the authors discussed CASIA and UBIRIS databases and describe the major characteristics of these common public and freely available noisy iris images databases (see Figure 2)

\subsection{CASIA Database [9]}

CASIA Iris Image Database (CASIA-Iris) developed by Chinese Academy of Sciences Institute of Automation research group (2004). This database has been released to the international biometrics community and updated from CASIA-IrisV1 to CASIA-IrisV4 since 2002. More than 3,000 users from 70 countries or regions have downloaded CASIAIris and much excellent work on iris recognition has been done based on these iris image databases.

CASIA Iris Image Database Version 1.0 (or CASIA Iris-V1) is probably the first iris image database publicly available to iris recognition researchers and has been widely used. It includes 756 iris images from 108 eyes, hence 108 classes. For each eye, 7 images are captured in two sessions, where three samples are collected in the first and four in the second session. Its images were captured within a highly constrained capturing environment, which conditioned the characteristics of the resultant images. They present very close and homogeneous characteristics, and their noise factors are exclusively related with iris obstructions by eyelids and eyelashes.

CASIA Iris Image Database Version 2.0 (CASIA IrisV2) includes two subsets captured with two different devices: Irispass-h developed by OKI and self-developed device

\begin{tabular}{|c|c|c|}
\hline \multicolumn{3}{|c|}{ Public Noisy Iris Image Databases } \\
\hline \multirow{6}{*}{$\frac{\infty}{\frac{0}{3}}$} & & CASIA- IrisV1 \\
\hline & \multicolumn{2}{|r|}{ CASIA- IrisV2 } \\
\hline & \multirow{3}{*}{ 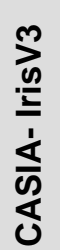 } & CASIA- IrisV3-Interval \\
\hline & & CASIA- IrisV3 -Lamp \\
\hline & & CASIA- IrisV3-Twins \\
\hline & \multicolumn{2}{|r|}{ CASIA- IrisV4 } \\
\hline \multirow{2}{*}{$\frac{\frac{\omega}{\tilde{\alpha}}}{\frac{\mathscr{m}}{丂}}$} & \multicolumn{2}{|r|}{ UBIRIS.v1 } \\
\hline & \multicolumn{2}{|r|}{ UBIRIS.v2 } \\
\hline
\end{tabular}
CASIA-IrisCamV2. Each subset includes 1200 images from 60 classes.

Figure 2 Public Noisy Iris Image Databases

CASIA-IrisV3 includes three subsets which are labelled as CASIA-IrisV3-Interval, CASIA-IrisV3-Lamp, CASIAIrisV3-Twins. CASIA-IrisV3 contains a total of 22,035 iris images from more than 700 subjects. All iris images are 8 bit gray-level JPEG files, collected under near infrared illumination. Almost all subjects are Chinese except a few in CASIA-IrisV3-Intervals. Because the three data sets were collected in different times, only CASIA-IrisV3-Interval and CASIA-IrisV3-Lamp have a small overlap in subjects. This database is used by many researchers to investigate the performance of their algorithms. 
CASIA-IrisV4 is an extension of CASIA-IrisV3 and contains six subsets. The three subsets from CASIA-IrisV3 are CASIA-Iris-Interval, CASIA-Iris-Lamp, and CASIA IrisTwins respectively. The three new subsets are CASIA IrisDistance, CASIA-Iris-Thousand, and CASIA-Iris-Syn. CASIA-IrisV4 contains a total of 54,601 iris images from more than 1,800 genuine subjects and 1,000 virtual subjects. All iris images are 8 bit gray-level JPEG files, collected under near infrared illumination or synthesized. The six data sets were collected or synthesized at different times and CASIA Iris-Interval, CASIA-Iris-Lamp, CASIA-Iris-Distance,CASIA Iris-Thousand may have a small inter-subset overlap in subjects.

\subsection{UBIRIS Database}

The UBIRIS database has two distinct versions: UBIRIS Version -1 (or UBIRIS.v1) [10] is composed of 1877 images collected from 241 persons during September, 2004. The most relevant characteristic of UBIRIS.v1 is to incorporate images with several noise factors, simulating less constrained image acquisition environments. The images stored in this database were captured by Nikon E5700 with configuration such as software E5700v1.0, format JPEG with RGB color representation, focal length $71 \mathrm{~mm}$ and exposure time $1 / 30 \mathrm{sec}$. It is public and freely available.

UBIRIS Version -2 (or UBIRIS.v2) [11] is another version of the UBIRIS database has over 11,000 images. Images stored in this database were actually captured on non-constrained conditions (at-a-distance, on-the-move and on the visible wavelength), with corresponding more realistic noise factors. Here, the images were captured by Canon EOS 5D with configuration such as sRGB color representation, shutter speed 1/197 sec. and exposure time 1/200 sec. It is public and freely available.

\section{PERFORMANCE EVALUATION}

In the proposed segmentation technique, the authors start their work implementation by K-means clustering to get the iris region; then delete small blocks and noise; followed by vertical Canny Edge detection for iris region; then Circular Hough transformation to determine iris; remove noise (upper and lower eyelid luminance) and localize $\&$ remove pupil (see Figure 3).

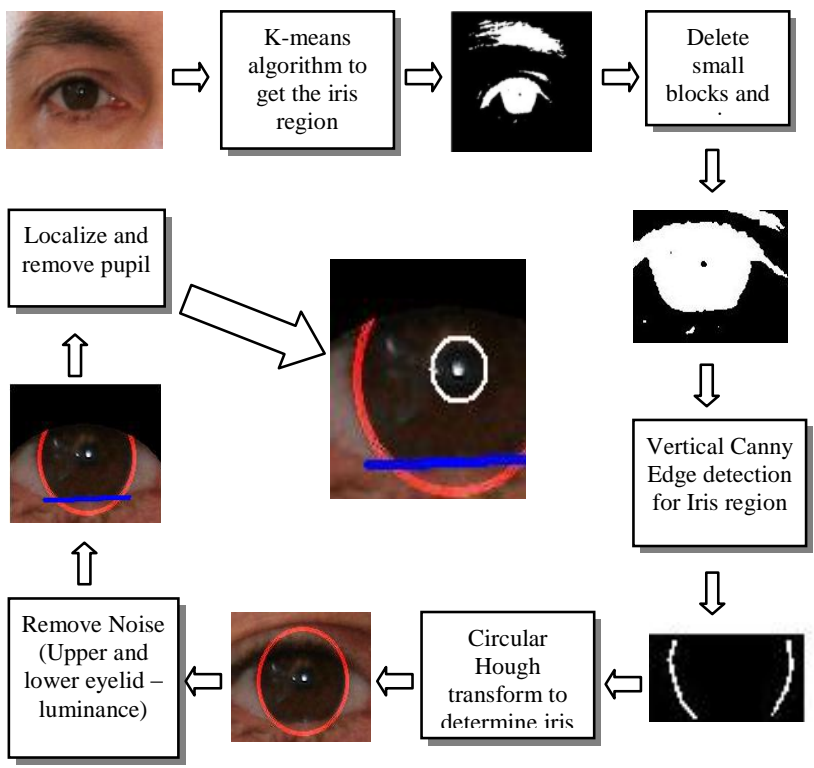

Figure 3 Block diagram of proposed iris segmentation method [1]
The authors assume that both the iris and the pupil have circular form and described by the values of its center $(x, y)$ and its radius $r$. Figure 4 shows the segmented images after implementation of proposed iris segmentation technique using a dataset of UBIRIS.v1 and UBIRIS.v2. After implementation of proposed iris segmentation technique, the authors compute the accuracy of the resultant images.
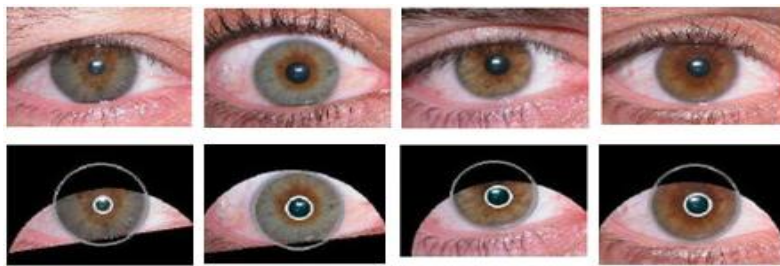

Figure 4 Noisy Iris Images with their Segmented Version

Table 1 shows the performance of proposed iris segmentation technique with some existing techniques for noisy iris images in terms of Accuracy and Time. As the results shown, the accuracy of the proposed technique is better than Daugman, Camus-Wildes and Martin-Roche techniques. At the same time the execution time of proposed segmentation technique is the lowest one i.e. 1.47 seconds.

Table 1 Performance Evaluation of Proposed Segmentation Algorithm with existing techniques for noisy iris images in terms of Accuracy and Time

\begin{tabular}{|c|c|c|}
\hline $\begin{array}{c}\text { Iris Segmentation Method } \\
\text { for Noisy Image }\end{array}$ & Accuracy & Time(s) \\
\hline Daugman & $95.22 \%$ & 2.73 \\
\hline Camus and Wildes & $98.68 \%$ & 1.95 \\
\hline Martin-Roche & $77.18 \%$ & 2.91 \\
\hline Proposed Technique & $\mathbf{9 8 . 7 2 \%}$ & $\mathbf{1 . 4 7}$ \\
\hline
\end{tabular}

Performance of proposed iris segmentation is also evaluated by using Equal Error Rate (EER). EER in iris recognition system is the point at which False Matching Rate (FMR) and False Non-Match Rate (FNMR) are almost equal. When the proposed iris segmentation technique is applied in an iris recognition system, the EER is very low i.e. 0.117 (see Figure 5) as compared with EER, when Daugman segmentation technique is used. This result shows that the proposed segmentation technique accurately isolate error regions in iris template.

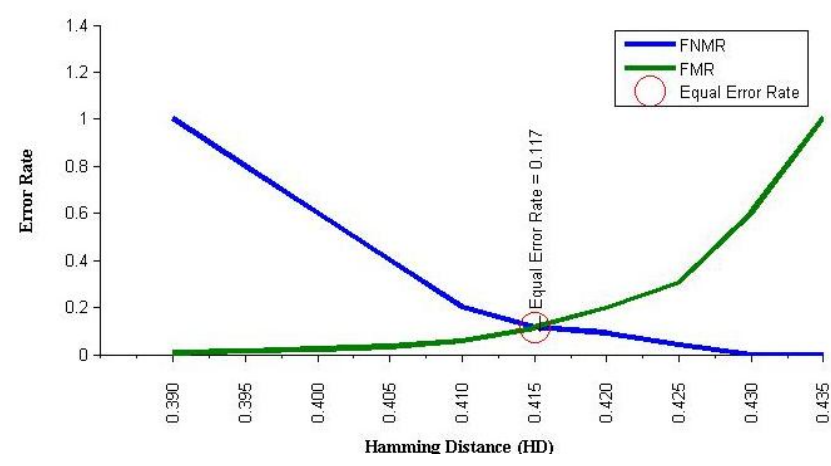

Figure 5 Equal Error Rate (EER) when the proposed iris segmentation technique is used 
In iris recognition system, to draw the match and nonmatch distributions, each iris image in the database is compared with all other available irises. After noise regions are discounted, Hamming Distance (HD) is a partial gauge/unit of variation Figure 6 shows the distribution of the hamming distance when the proposed iris segmentation technique is used and Figure 7 shows the distribution of the hamming distance when Daugman segmentation is used.

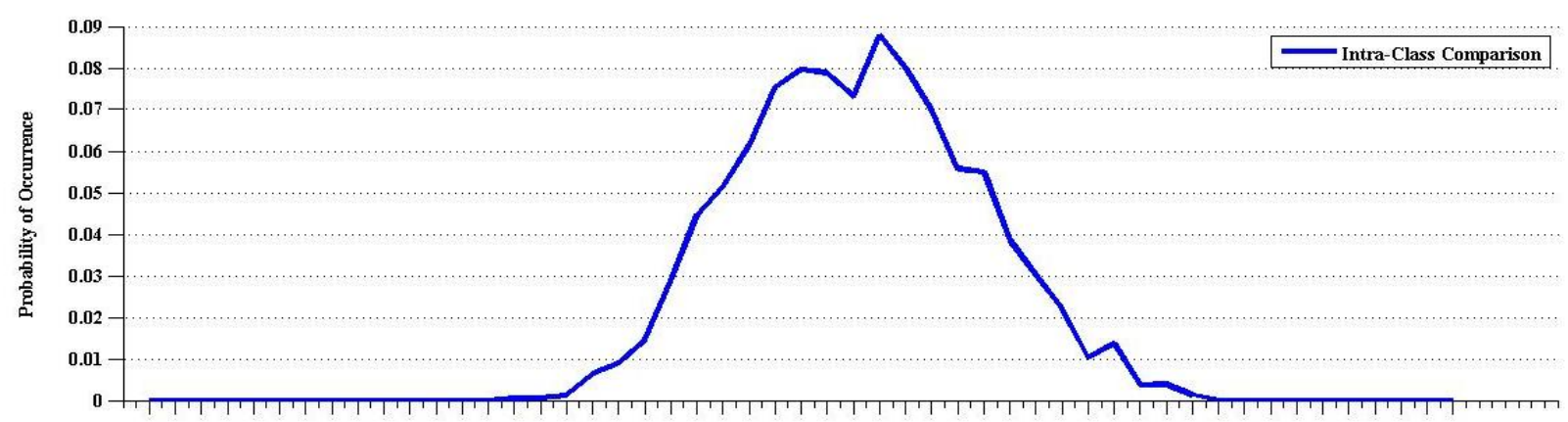

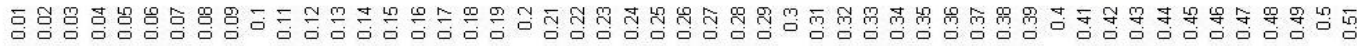

Hamming Distance (HD)

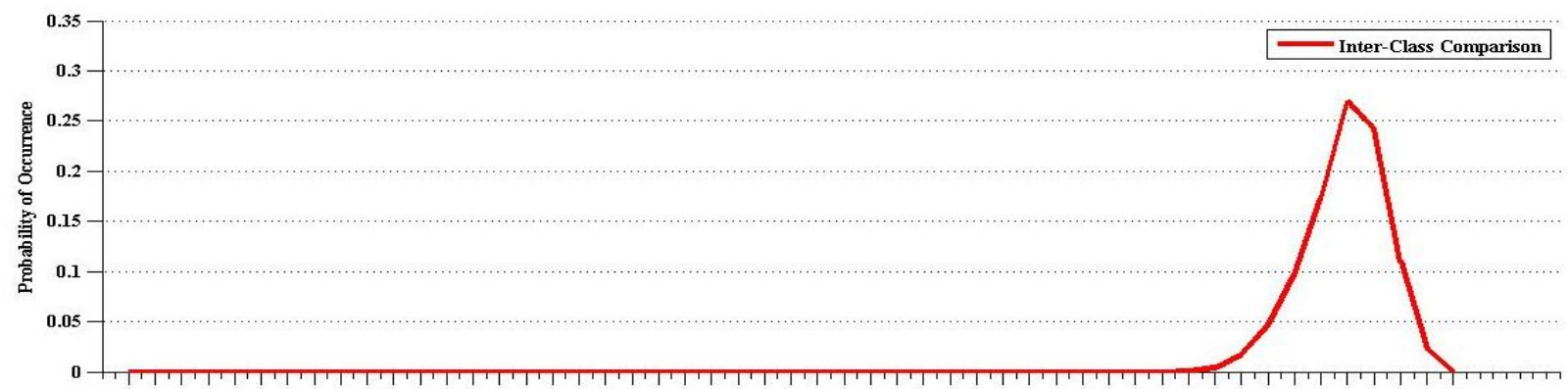

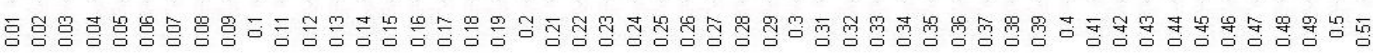

Hamming Distance (HD)

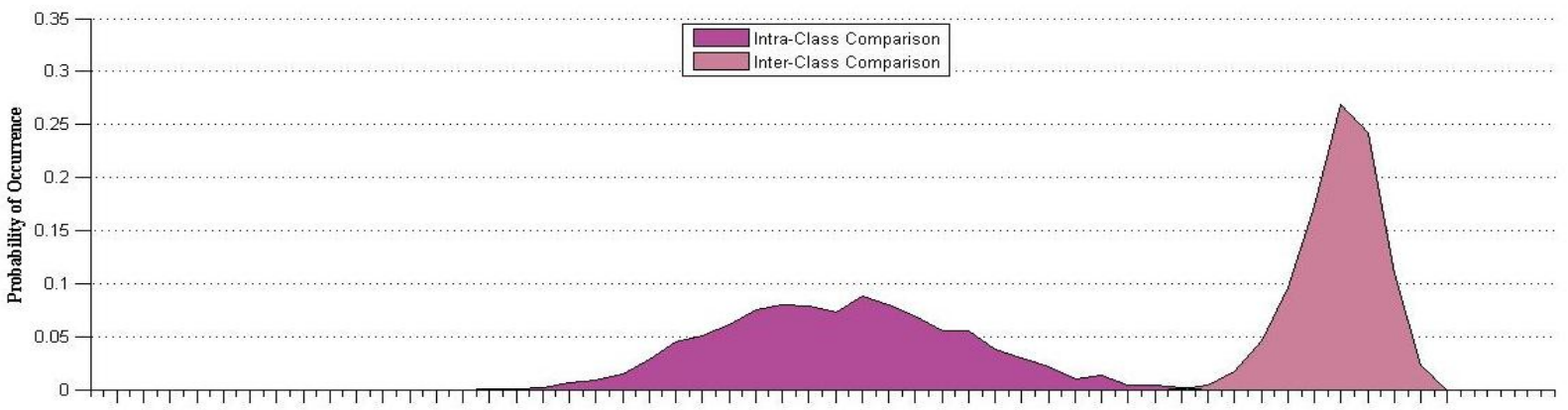

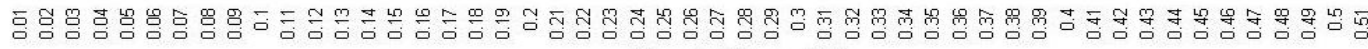

Hamming Distance (HD)

Figure 6 the match and non-match distributions when Proposed Iris Segmentation Technique is used

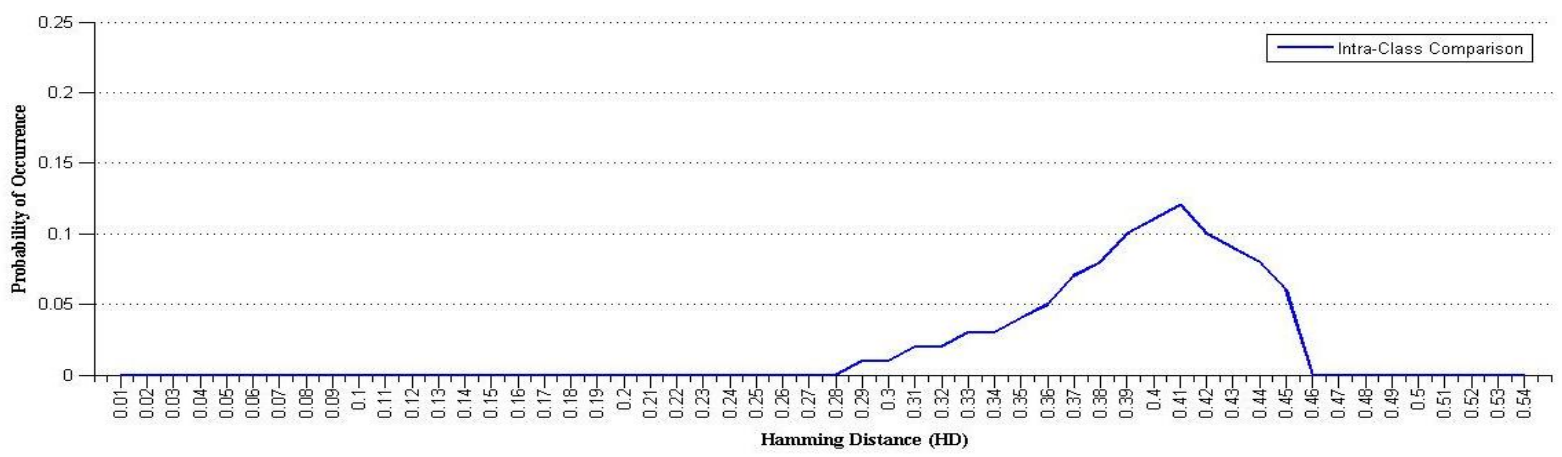



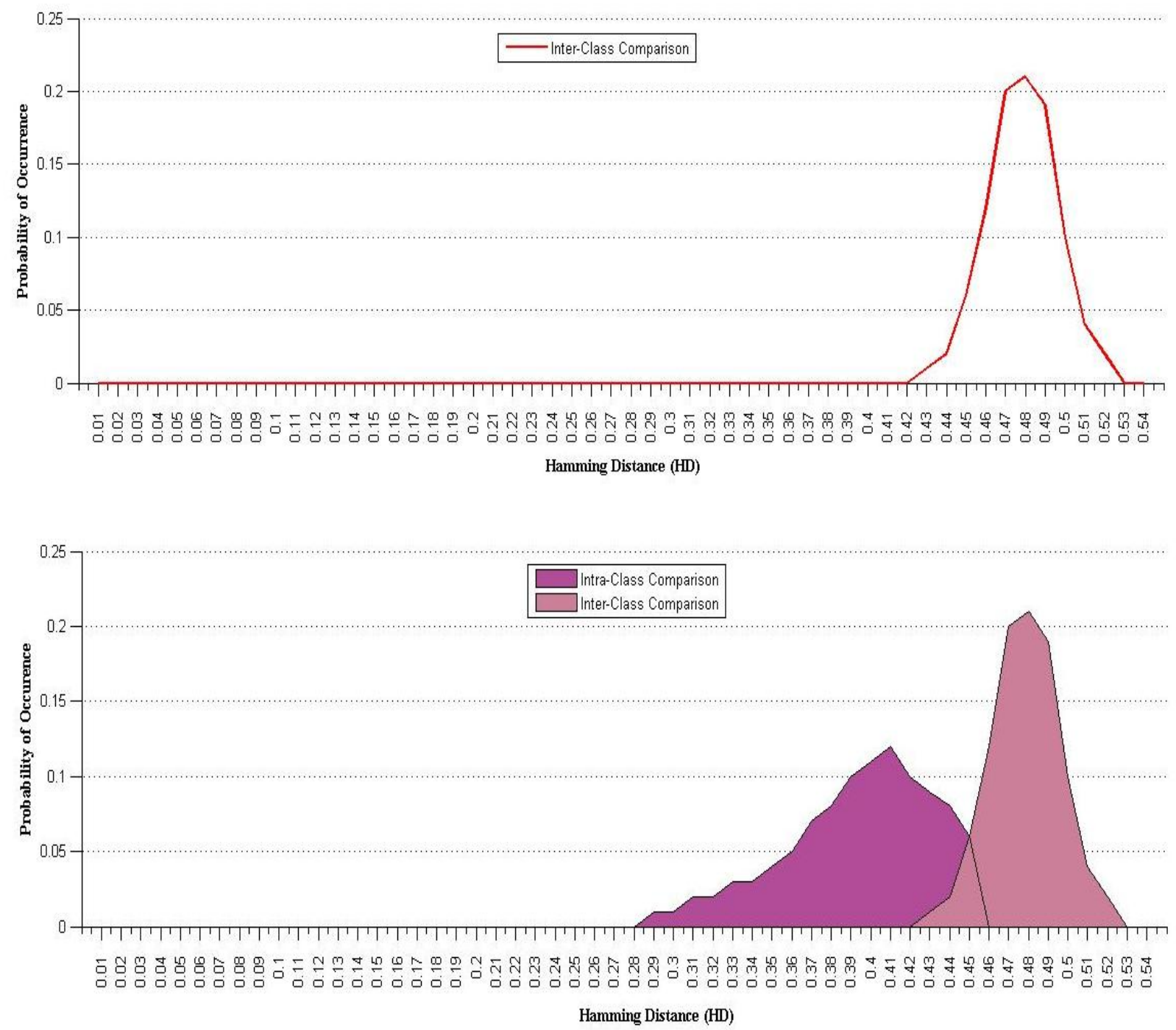

Figure 7 the match and non-match distributions when Daugman Iris Segmentation Technique is used

\section{CONCLUSION AND FUTURE SCOPE}

This paper compares the performance of a number of iris segmentation techniques, emphasizing the accuracy and time as well as equal error rate. Iris Recognition technology provides a practically \& significantly feasible technique for overcoming the performance and user acceptability obstacles to the widespread adoption of biometric systems. Much research effort around the world is being applied for expanding the accuracy and capabilities of this biometric domain, with a consequent broadening of its application in the near future. This research paper shows the outcome of the performance evaluation of the proposed effective iris segmentation technique for noisy iris images. As per the results received after implementation, the proposed technique shows the $98.72 \%$ accuracy and at the same time the execution time of the proposed iris segmentation technique is 1.47 seconds. The Equal Error Rate (EER) of the proposed iris segmentation technique is very low i.e. 0.117.

The authors future work will concern segmenting noisy irises when the lower or upper eyelids and eyelashes cover the pupil of the iris, which is currently not handled and optimization of the code, so that the segmentation software can run in real time applications.
6. REFERENCES

[1] Rajeev Gupta, Ashok Kumar, Implementation of Proposed Effective Segmentation Technique for Noisy Iris Images, 2014, International Journal of Advanced Information Science and Technology, vol. 29(29), pp. 69-74

[2] Rajeev Gupta, Ashok Kumar, An Effective Segmentation Technique for Noisy Iris Images, 2013, International Journal of Application or Innovation in Engineering \& Management, vol. 2(12), pp. 118-125

[3] Daugman, J, 1993, High confidence visual recognition of persons by a test of statistical independence, IEEE Transactions on Pattern Analysis an. Machine Intelligence., vol. 15(11), pp. 1148-1161

[4] Camus, T.A., and Wildes, R, 2004, Reliable and fast eye finding in closeup images. IEEE 16th International Conference on Pattern Recognition, Quebec, Canada, pp. 389-394

[5] Proenca, H., 2006, Towards Non-Cooperative Biometric Iris Recognition. $\mathrm{PhD}$ thesis, University of Beira Interior. 
[6] Proenca, H., 2010, Iris Recognition: On the Segmentation of Degraded Images Acquired in the Visible Wavelength, IEEE Transactions on Pattern Analysis and Machine Intelligence, vol. 32(8), pp. 1502-1516.

[7] Martin-Roche, D., Sanchez-Avila, C., and SanchezReillo, R.,2002, Iris recognition for biometric identification using dyadic wavelet transform zero-crossing, IEEE Aerospace and Electronic Systems Magazine, vol. 17(10), pp. 3-6

[8] Dobes, M., Martineka, J., Dobes, D.S.Z., Pospisil, J., 2006, Human Eye Localization Using the Modified Hough Transform, Optik, vol. 117(10), pp. 468-473.

[9] CASIA Iris Image Database, http://biometrics.idealtest.org/
[10] Proenca, H. and Alexandre, L. A., 2005, UBIRIS: A noisy iris image database, In Proceedings of the 13th International Conference on Image Analysis and Processing, pp. 970-977.

[11] Proença, H., Filipe, S, Santos, R, Oliveira, J, Alexandre, L.A., 2010, The UBIRIS.v2: A Database of Visible Wavelength Iris Images Captured On-The-Move and At-A-Distance, IEEE Transactions on Pattern Analysis and Machine Intelligence, vol. 32(8), pp. $1529-1535$

[12] Rajeev Gupta, Ashok Kumar, Noisy Iris Recognition \& its importance, 2013, Journal of Ultra Scientist of Physical Sciences International Journal of Physical Sciences, vol. 25(2)B, pp. 229-234 